\title{
1 Super-infections and relapses occur in chronic norovirus infections
}

2 Julianne R Brown ${ }^{1}$, Sunando Roy ${ }^{2}$, Helena Tutill ${ }^{2}$, Rachel Williams ${ }^{2}$, Judith Breuer ${ }^{1,2}$

3

41 Microbiology, Virology and Infection Prevention and Control, Great Ormond Street Hospital for

$5 \quad$ Children NHS Foundation Trust

$6 \quad 2$ Infection and Immunity, University College London, UK

7

8 Running title: Norovirus super-infections

$9 \quad$ Abstract word count: 241

10 Text word count: 1317

11 Corresponding author

12 Julianne R Brown

13 Julianne.brown@nhs.net

$14 \quad 00442074059200$ Ext 5929

15 
Norovirus causes chronic infections in immunocompromised patients with considerable associated morbidity. It is not known whether chronic infections involve super- or re-infections or relapses.

\section{Objectives}

21 To retrospectively investigate whether longitudinal sampling in chronically infected patients demonstrates persistent infection with the same virus, or super- or re-infection.

\section{Study design}

24 Norovirus full genomes were generated from 86 longitudinal samples from 25 paediatric patients. Consensus sequences were used for phylogenetic analysis and genotyping.

\section{Results}

27 Super-infections occurred in $17 \%$ of chronically infected patients who were continuously PCR positive; including two with mixed norovirus infections. The median duration of infection was 107 days longer in those with super-infections; however this was not statistically significant. A third of patients with interrupted norovirus shedding continued to be infected with the same virus despite up to 2 months of PCR negative stools, classified as a relapse. The majority $(67 \%)$ of patients with interrupted shedding were re-infected with a different genotype.

\section{Conclusions}

34 Chronically infected patients who are continuously PCR positive are most likely to remain infected with the same virus; however super-infections do occur leading to mixed infection. Patients with interrupted shedding are likely to represent re-infection with a different genotype, however

37 relapsing infections also occur. 
38 Our findings have implications for infection control as immunosuppressed patients remain

39 susceptible to new norovirus infections despite current or recent infection and may continue to be

40 infectious after norovirus is undetectable in stool. The relevance to children without co-morbidities

41 remains to be determined.

\section{$42 \quad$ Highlights}

43 - Super-infections occur in $17 \%$ of continuously shedding chronic norovirus infections

44 - Re-infections occur in two-thirds of chronic infections with interrupted shedding

- Relapses occur in a third of chronic infections with interrupted shedding

- Patients are susceptible to super- and re-infection with different genotypes

\section{$47 \quad$ Keywords}

48 Norovirus; chronic; re-infection; super-infection; relapse; full genomes 
Norovirus is a leading cause of gastroenteritis. Infections are typically self-limiting in immunocompetent hosts, with limited morbidity aside from dehydration. In immunocompromised patients however, there is a risk of chronic infection with significant associated morbidity [1]. Chronic infections are bi-phasic with an acute phase of vomiting and diarrhoea, followed by chronic viral shedding and diarrhoea lasting weeks to years. The majority of case reports describe patients to be symptomatic during this extended period of shedding, with up to 24 bowel movements per day [2]. However chronic infections can experience intermittent symptoms of diarrhoea [3] or be asymptomatic [4].

The Norovirus genus is comprised of five genogroups (GI-GV), of which GI, GIl and, to a limited extent, GIV cause infections in humans. Each genogroup is further classified into genotypes; GI.1-9 and GII.1-22. GII.4 genotypes, which are the predominant global genotype since the mid-1990s [5], are divided into variant types. Norovirus has a dual typing system based on the polymerase (ORF1) and capsid (ORF2) sequences.

\section{Objectives}

We retrospectively sequence full norovirus genomes from longitudinally sampled chronic infections for genotyping and phylogenetic analysis, to determine whether patients remain persistently infected with the same strain or whether super- or re-infections occur.

\section{Study Design}

Eighty-six longitudinal stool samples were retrospectively sequenced from 25 paediatric patients, with two to eight samples per patient. Samples were collected between November 2012 and January 2016 from patients with persistent norovirus infections (PCR positive $>1$ month) for whom two or more longitudinal stool specimens were available. Patients were under the care of a UK paediatric tertiary referral hospital. Norovirus positive patients were tested weekly whilst inpatients or monthly whilst outpatients for the presence or absence of norovirus by the diagnostic Virology 
laboratory using a reverse-transcriptase real-time multiplex PCR to detect norovirus GI and GII, the methods for which are described elsewhere [6].

Of the 25 patients, 18 were continuously norovirus positive (continuous shedding), with a median of 129 days between the first and last sequenced sample (range 7-466). A further seven patients had a period between the first and last sequenced sample during which norovirus was not detected in stool, before once again being detected (interrupted shedding). In addition two of the 18 patients who shed norovirus continuously (Patient 63 and 73), proceeded to become norovirus PCR negative following which both became positive again. In total nine patients had interrupted norovirus shedding (median 153 days undetected, range 9-466).

Norovirus genome sequencing and phylogenetic analysis are described in Supplementary Methods.

Results

Continuously positive patients

Of the 18 patients who were continuously norovirus PCR positive, 15/18 (83\%) remained infected with the same genotype throughout the study period, classified as persistent infections (Table 1). The longitudinal samples from each of these patients cluster together on the phylogenetic tree (Figure 1), indicating these patients remained infected not only with the same genotype but with the same virus. Three of the 18 (17\%) patients with continuous shedding had evidence of infection with a second genotype occurring during the study period (Patients 73, 65 and 101), classified as super-infections. Super-infection is proven for two patients (Patient 65 and Patient 101) in whom co-infection with two different genotypes was detected in a single sample. Patient 73 was initially infected with a GII.Pe_GII.4 virus then became infected with GII.P16_GII.17, although a mixture of the two genotypes in the same sample was not detected. Patient 73 was continually positive for norovirus in stool; the interval between detection of GII.Pe_GII.4 and of GII.P16_GII.17 was 22 days with an 
additional positive stool sample taken during this interval (not available for sequencing). We cannot confirm whether Patient 73 cleared GII.Pe_GII.4 prior to infection with GII.P16_GII.17 or whether a temporary mixed infection occurred, however given the short interval between positive PCR tests (1-2 weeks), the latter is most probable.

These data suggest that in patients who are continuously norovirus PCR positive, super-infection occurs in a sixth (17\%) of cases. The median duration of infection was 322 days (range 58-738 days) in the three patients who had a super-infection and 215 days (range 14-711 days) in the 15 who did not. The duration of infection was not significantly different $(P=0.360)$.

\section{Patients with interrupted norovirus shedding}

Of the nine patients who become norovirus PCR negative and then positive again, five (Patients 34 , $68,73,147$ and 176) acquired a second virus with a different genotype to the first, classified as reinfection (Table 1). For Patient 73 this was the second incidence of re-infection, the first having occurred whilst continuously norovirus PCR positive (Supplementary Figure 1).

Another of the nine patients, Patient 63, appeared to be infected with the same genotype (GII.P21_GII.3) after a period of 466 days during which norovirus was undetectable by PCR. Phylogenetic analysis revealed the second virus to be a different variant of GII.P21_GII.3, since the sequences from before and after the PCR negative period do not cluster together (Figure 1).

Thus the majority $(6 / 9,67 \%)$ of patients with interrupted norovirus shedding had been re-infected with a different genotype or variant.

For the remaining three patients (Patients 31, 72 and 75), the second virus was of the same genotype, clustering with the earlier virus in the phylogenetic analysis tree (Figure 1), classified as relapse. This suggests cryptogenic persistence of the first virus. The three relapse patients had the shortest intervals during which norovirus was undetectable; less than two months compared to 2-15 months for those who were re-infected with a new genotype or variant. 
123 Excluding those patients with mixed infections, in the patients who were continually infected with the same virus there was a strong positive correlation between the number of consensus sequence pairwise single nucleotide variants (SNVs) and the number of days separating specimen collection $\left(\mathrm{R}^{2}\right.$ $0.775, \mathrm{P}<0.001$ ) (Supplementary Figure 2 ) with up to 131 SNVs accumulating across the genome over 445 days.

\section{Discussion}

We use full genome sequencing to show that super-infection and re-infection occurs in patients in whom norovirus can be detected over long periods. When the virus is shed continuously, superinfection was detected in a sixth (17\%) of patients while re-infections accounted for the majority (67\%) of cases where norovirus was detected after interrupted shedding. Whether a lack of protection against super- and re-infection extends to children without comorbidities remains to be determined.

Conversely, relapse was identified in patients in whom norovirus shedding was interrupted for up to two months. These data may have implications for clinical practice; chronically infected patients who appear to clear norovirus may still harbour persistent but undetectable virus. Whether or not these patients present a transmission risk is not known. However, a prudent course of action would be to consider immunosuppressed patients who have cleared virus after a chronic infection as potentially infectious for up to 2-3 months following the last positive stool and to continue PCR surveillance for this period. Given the small sample size in this study (three patients relapsing) a larger study is required to confirm these findings.

Our data confirms previous observations that viruses persistently infecting immunocompromised patients are continuously mutating, leading to the accumulation of SNVs $[3,7]$. The resulting intra- 

be a reservoir for the emergence of novel viral variants $[7,8]$, however the estimated rarity of such

147 events has led to the conclusion that immunosuppressed hosts are not the principle source of novel 148 variants at the epidemiological scale [9].

149 Mixtures of norovirus strains have been detected in individuals in oyster-borne norovirus outbreaks $150[10,11]$; to our knowledge this is the first identification of mixed genotypes within a single host in 151 sporadic infections. Co-infecting norovirus strains within an individual provides the opportunity for 152 viral recombination to occur, a feature that is known to be important in norovirus evolution and has 153 been suggested to contribute to the emergence of new pandemic strains [12]. 


\section{Figure titles}

Table 1. Summary of longitudinally sampled norovirus infections. The occurrence of re-infection is inferred from phylogenetic analysis of norovirus full genome sequences

Figure 1. Full genome maximum likelihood phylogeny of longitudinal norovirus sequences.

Sequences are labelled with a unique patient number $(\mathrm{Px}, \mathrm{NORO} / \mathrm{XX})$ and serial longitudinal numbering (e.g. NORO/XX-1). The node shape and colour indicates whether the position on the tree suggests persistence of the same virus, re-infection with a different genotype or re-infection with a different strain of the same genotype. Co-infections with multiple genotypes (Patient 65 and 101) are not shown since reliable consensus sequences for phylogenetic analysis cannot be generated. Footnote: Longitudinal samples from Patients 58 and 63 (63-1 and 63-2) do not cluster as closely together as longitudinal samples from other patients; 445 and 135 days had passed between the longitudinal samples therefore is consistent with accumulation of mutations over time. The second sample from patient 58 clusters closely with samples from patient 68 and the early samples from Patient 63 cluster with samples from Patient 61; these patients were epidemiologically linked (data not shown).

Supplementary Figure 1. Timeline of norovirus detection in Patient 73. Orange markers indicate detection of norovirus GII.Pe_GII.4, blue detection of GII.P16_GII.17 and green detection of GII.P21_GII.3. Grey markers indicate norovirus positive samples that were not availbale for sequencing. Black markers with a Ct value of zero indicate norovirus was not detected by RT-qPCR.

Supplementary Figure 2. Number of intra-host pairwise single nucleotide variants (SNVs) across norovirus full genomes in longitudinally sampled norovirus infections, plotted against number of days separating the samples. 


\section{Conflict of interest statement}

The authors declare no conflict of interest. The funders had no role in study design, data collection and interpretation, or the decision to submit the work for publication.

\section{Funding statement}

This work was supported by the PATHSEEK European Union's Seventh Programme for research, technological development and demonstration (grant number 304875) and a National Institute for Health Research (NIHR) doctoral fellowship (grant number NIHR-HCS-D12-03-15) to JRB. JBreuer receives funding from the NIHR UCL/UCLH biomedical research centre (BRC).

\section{Acknowledgments}

We acknowledge the infrastructure support from the UCL Pathogen Genomics Unit (PGU), the NIHR UCL/UCLH BRC, the UCL MRC CMMV and the Great Ormond Street Hospital for Children NHS Foundation Trust Microbiology, Virology and Infection Prevention and Control department. All research at Great Ormond Street Hospital NHS Foundation Trust is made possible by the NIHR Great Ormond Street Hospital Biomedical Research Centre. The views expressed are those of the author(s) and not necessarily those of the NHS, the NIHR or the Department of Health.

\section{References}

[1] Green KY. Norovirus infection in immunocompromised hosts. Clin Microbiol Infect. 2014;20:71723.

[2] Ghosh N, Malik FA, Daver RG, Vanichanan J, Okhuysen PC. Viral associated diarrhea in immunocompromised and cancer patients at a large comprehensive cancer center: a 10-year retrospective study. Infectious diseases (London, England). 2016:1-7.

[3] Schorn R, Hohne M, Meerbach A, Bossart W, Wuthrich RP, Schreier E, et al. Chronic norovirus infection after kidney transplantation: molecular evidence for immune-driven viral evolution. Clinical infectious diseases : an official publication of the Infectious Diseases Society of America.

2010;51:307-14.

[4] Westhoff TH, Vergoulidou M, Loddenkemper C, Schwartz S, Hofmann J, Schneider T, et al. Chronic norovirus infection in renal transplant recipients. Nephrology, dialysis, transplantation : official publication of the European Dialysis and Transplant Association - European Renal Association. 2009;24:1051-3.

[5] Vinje J. Advances in Laboratory Methods for Detection and Typing of Norovirus. Journal of clinical microbiology. 2014. 
[6] Brown JR, Shah D, Breuer J. Viral gastrointestinal infections and norovirus genotypes in a paediatric UK hospital, 2014-2015. Journal of clinical virology : the official publication of the Pan American Society for Clinical Virology. 2016;84:1-6.

[7] Bull RA, Eden JS, Luciani F, McElroy K, Rawlinson WD, White PA. Contribution of intra- and interhost dynamics to norovirus evolution. Journal of virology. 2012;86:3219-29.

[8] Sukhrie FH, Siebenga JJ, Beersma MF, Koopmans M. Chronic shedders as reservoir for nosocomial transmission of norovirus. Journal of clinical microbiology. 2010;48:4303-5.

[9] Eden JS, Chisholm RH, Bull RA, White PA, Holmes EC, Tanaka MM. Persistent infections in immunocompromised hosts are rarely sources of new pathogen variants. Virus evolution.

2017;3:vex018.

[10] Wang Y, Zhang J, Shen Z. The impact of calicivirus mixed infection in an oyster-associated outbreak during a food festival. Journal of clinical virology : the official publication of the Pan American Society for Clinical Virology. 2015;73:55-63.

[11] Symes SJ, Gunesekere IC, Marshall JA, Wright PJ. Norovirus mixed infection in an oysterassociated outbreak: an opportunity for recombination. Archives of virology. 2007;152:1075-86.

[12] Eden JS, Tanaka MM, Boni MF, Rawlinson WD, White PA. Recombination within the pandemic norovirus GII.4 lineage. Journal of virology. 2013;87:6270-82. 